\title{
ON MODULAR SIGNS
}

\author{
E. KOWALSKI, Y.-K. LAU, K. SOUNDARARAJAN \& J. WU
}

\begin{abstract}
We consider some questions related to the signs of Hecke eigenvalues or Fourier coefficients of classical modular forms. One problem is to determine to what extent those signs, for suitable sets of primes, determine uniquely the modular form, and we give both individual and statistical results. The second problem, which has been considered by a number of authors, is to determine the size, in terms of the conductor and weight, of the first sign-change of Hecke eigenvalues. Here we improve the recent estimate of Iwaniec, Kohnen and Sengupta.
\end{abstract}

\section{INTRODUCTION}

There are many results in the arithmetic of modular forms which are concerned with various ways of characterizing a given primitive cusp form $f$ from its siblings, starting from the fact that Fourier coefficients, hence the $L$-function, determine uniquely a cusp form $f$ relative to a congruence subgroup $\Gamma$ of $S L(2, \mathbb{Z})$. Among such results are stronger forms of the multiplicity one theorem for automorphic forms or representations, various explicit forms of these statements, where only finitely many coefficients are required (say at primes $p \leqslant X$, for some explicit $X$ depending on the parameters defining $f$ ), and a number of interesting "statistic" versions of the last problem, where $X$ can be reduced drastically, provided one accepts some possible exceptions. Among other papers, we can cite [4], [16], [3] or [9].

Some of these statements were strongly suggested by the analogy with the problem of the least quadratic non-residue, which is a problem of great historic importance in analytic number theory, and there are many parallels between the results which have been obtained. However, this parallel breaks down sometimes. For instance, in [13], Lau and $\mathrm{Wu}$ note that one result of [12] for the least quadratic non-residue is highly unlikely to have a good analogue for modular forms. This result (see [12, Th. 3]) is a precise estimate for the number of primitive real Dirichlet characters of modulus $q \leqslant D$ for which the least $n$ with $\chi(n)=-1$ is $\gg \log D$, and the difficulty is that this estimate can be understood by assuming that the values $\chi(p)$, for $p$ of moderate size compared with $D$, behave like independent random variables taking values \pm 1 equally often. However, Hecke eigenvalues may take many more than two values, and thus assuming that they coincide should definitely be a much more stringent condition.

Date: November 2, 2018.

2000 Mathematics Subject Classification. 11F30, 11F41, 11K36, 11 N35.

Key words and phrases. Fourier coefficients of modular forms, Hecke eigenvalues, RankinSelberg convolution, symmetric powers, sieve methods, equidistribution, Sato-Tate conjecture.

The work of E. Kowalski was supported in part by the National Science Foundation under agreement No. DMS-0635607 during a sabbatical stay at the Institute for Advanced Study.

The work of K. Soundararajan was partially supported by the National Science Foundation (DMS 0500711), and through the Veblen Fund of the Institute for Advanced Study, Princeton. 
In this paper, we consider a way to potentially recover a closer analogy: namely (narrowing our attention to forms with real eigenvalues) instead of looking at the values of the Hecke eigenvalues, we consider only their signs (where we view 0 as being of both signs simultaneously, to increase the possibility of having same sign). Then classical questions for Dirichlet characters and modular forms have the following analogues for signs of Hecke eigenvalues $\lambda_{f}(p)$ of a classical modular form $f:$

- What is the first sign-change, i.e., the smallest $n \geqslant 1$ (or prime $p$ ) for which $\lambda_{f}(n)<0$ (or $\left.\lambda_{f}(p)<0\right)$ ? (Analogues of the least quadratic non-residue). Note a small difference with quadratic characters: it is not true here that the smallest integer with negative Hecke eigenvalue is necessarily prime; finding one or the other are two different questions. ${ }^{1}$

- Given arbitrary signs $\varepsilon_{p} \in\{ \pm 1\}$ for all primes, what is the number of $f$ (in a suitable family) for which $\lambda_{f}(p)$ has $\operatorname{sign} \varepsilon_{p}$ for all $p \leqslant X$, for various values of $X$ ? (Analogue of the question in [12]).

- In particular, is there a finite limit $X$ such that coincidences of signs of $\lambda_{f}(p)$ and $\varepsilon_{p}$ for all $p \leqslant X$ implies that $f$ is uniquely determined? (Analogue of the multiplicity one theorem).

Of these three problems, only the first one seems to have be considered earlier, with the best current result due to Iwaniec, Kohnen and Sengupta [6]. We will improve it, and obtain some first results concerning the other two problems. We will also suggest further questions that may be of interest.

Before stating our main theorems, here are the basic notation about modular forms (see, e.g., [7, Ch. 14] for a survey of these facts). We denote by $\mathrm{H}_{k}^{*}(N)$ the finite set of all primitive forms of weight $k$ for $\Gamma_{0}(N)$, where $k \geqslant 2$ is an even integer and $N \geqslant 1$ is an integer. The restriction to trivial Nebentypus ensures that all Fourier coefficients are real, and for any $f \in \mathrm{H}_{k}^{*}(N)$, we denote

$$
f(z)=\sum_{n=1}^{\infty} \lambda_{f}(n) n^{(k-1) / 2} e(n z), \quad e(z)=e^{2 i \pi z}, \quad(\Im m z>0)
$$

its Fourier expansion at infinity. Since $f$ is primitive, the $\lambda_{f}(n)$ are the normalized eigenvalues of the Hecke operators $T_{n}$, and satisfy the well-known Hecke relations

$$
\lambda_{f}(m) \lambda_{f}(n)=\sum_{\substack{d \mid(m, n) \\(d, N)=1}} \lambda_{f}\left(\frac{m n}{d^{2}}\right)
$$

for all integers $m \geqslant 1$ and $n \geqslant 1$. In particular, $\lambda_{f}$ is a multiplicative function of $n$ (so $\lambda_{f}(1)=1$ ) and moreover the following important special case

$$
\lambda_{f}(p)^{2}=1+\lambda_{f}\left(p^{2}\right)
$$

holds for all primes $p \nmid N$.

Furthermore, it is also known that $\lambda_{f}(n)$ satisfies the deep inequality

$$
\left|\lambda_{f}(n)\right| \leqslant \tau(n)
$$

\footnotetext{
1 E.g., as a random example, for the cusp form of weight 2 associated to the elliptic curve $y^{2}=x^{3}+x$, the first negative coefficient is $\lambda(9)=-3$, and the first negative prime coefficient is $\lambda(13)=-6$.
} 
for all $n \geqslant 1$, where $\tau(n)$ is the divisor function (this is the Ramanujan-Petersson conjecture, proved by Deligne). In particular, we have $\lambda_{f}(p) \in[-2,2]$ for $p \nmid N$, and hence there exists a unique angle $\theta_{f}(p) \in[0, \pi]$ such that

$$
\lambda_{f}(p)=2 \cos \theta_{f}(p) .
$$

Our other notation is standard in analytic number theory: for instance, $\pi(x)$ denotes the number of primes $\leqslant x$ and $P^{+}(n)$ (resp. $P^{-}(n)$ ) denotes the largest (resp. smallest) prime factor of $n$, with the convention $P^{+}(1)=1$ (resp. $P^{-}(1)=$ $\infty)$.

We now describe our results.

1.1. The first negative Hecke eigenvalue. For $f \in \mathrm{H}_{k}^{*}(N), k \geqslant 2$ and $N \geqslant 1$, it is well-known that the coefficients $\lambda_{f}(n)$ change sign infinitely often. We denote by $n_{f}$ the smallest integer $n \geqslant 1$ such that $(n, N)=1$ and

$$
\lambda_{f}(n)<0 \text {. }
$$

The analogue (or one analogue) of the least-quadratic non-residue problem is to estimate $n_{f}$ in terms of the analytic conductor $Q:=k^{2} N$. Iwaniec, Kohnen and Sengupta [6] have shown recently that

$$
n_{f} \ll Q^{29 / 60}=\left(k^{2} N\right)^{29 / 60}
$$

(here, standard methods lead to $n_{f} \ll_{\varepsilon} Q^{1 / 2+\varepsilon}$, so the significance is that the exponent is $<1 / 2)$.

Our first result is a sharpening of this estimate:

Theorem 1. Let $k \geqslant 2$ be an even integer and $N \geqslant 1$. Then for all $f \in \mathrm{H}_{k}^{*}(N)$, we have

$$
n_{f} \ll Q^{9 / 20}=\left(k^{2} N\right)^{9 / 20},
$$

where the implied constant is absolute.

This bound is not the best that can be achieved using our method, and we will comment on this after its proof (in particular, an interesting function $\beta$ occurs when trying to push the idea to its limit).

One automatic improvement of the exponent arises from any subconvexity bound for the relevant $L$-functions, as already observed by Iwaniec, Kohnen and Sengupta. We do not need such deep results to prove Theorem 1, but we will state below the precise relation.

We do not know if the estimate of Theorem 1 holds for the first negative Hecke eigenvalue at a prime argument.

1.2. Statistic study of the first sign-change. The upper bound (1.6) is probably far from optimal. Indeed, one can show that under the Grand Riemann Hypothesis we have

$$
n_{f} \ll(\log (k N))^{2}
$$

where the implied constant is absolute. Our next result confirms this unconditionally for almost all $f$. It closely parallels the case of Dirichlet characters (see [12]). Precisely, we first recall that

$$
\left|\mathrm{H}_{k}^{*}(N)\right| \asymp k \varphi(N)
$$


where $\varphi(N)$ is the Euler function, as $k, N \rightarrow+\infty$, and we prove:

Theorem 2. Let $\nu \geqslant 1$ be a fixed integer and $\mathscr{P}$ be a set of prime numbers of positive density in the following sense:

$$
\sum_{\substack{z<p \leqslant 2 z \\ p \in \mathscr{P}}} \frac{1}{p} \geqslant \frac{\delta}{\log z} \quad\left(z \geqslant z_{0}\right)
$$

for some constants $\delta>0$ and $z_{0}>0$. Let $\left\{\varepsilon_{p}\right\}_{p \in \mathscr{P}}$ be a sequence of real numbers such that $\left|\varepsilon_{p}\right|=1$ for all $p$. Let $k \geqslant 2$ be an even integer and $N \geqslant 1$ be squarefree. Then there are two positive constants $C$ and $c$ such that the number of primitive cusp forms $f \in \mathrm{H}_{k}^{*}(N)$ satisfying

$$
\varepsilon_{p} \lambda_{f}\left(p^{\nu}\right)>0 \quad \text { for } \quad p \in \mathscr{P}, \quad p \nmid N \quad \text { and } \quad C \log (k N)<p \leqslant 2 C \log (k N)
$$

is bounded by

$$
\ll_{\nu, \mathscr{P}} k N \exp \left(-c \frac{\log k N}{\log \log k N}\right) .
$$

Here $C, c$ and the implied constant depend on $\nu$ and $\mathscr{P}$ only.

Taking $\mathscr{P}$ the set of all primes, $\varepsilon_{p}=1$ and $\nu=1$ in Theorem 2, we immediately get: ${ }^{2}$

Corollary 1. Let $k \geqslant 2$ be an even integer and $N \geqslant 1$ be squarefree. There is an absolute positive constant $c$ such that we have

$$
n_{f} \ll \log (k N),
$$

for all $f \in \mathrm{H}_{k}^{*}(N)$, except for $f$ in an exceptional set with

$$
\ll k N \exp \left(-c \frac{\log k N}{\log \log k N}\right)
$$

elements, where the implied constants are absolute.

It is very natural to ask whether this result is optimal (as the analogue is known to be for real Dirichlet characters). In this direction, we can prove the following:

Theorem 3. Let $N$ be a squarefree number and $k \geqslant 2$ an even integer, and let $\left(\varepsilon_{p}\right)$ be a sequence of signs indexed by prime numbers. For any $\varepsilon>0, \varepsilon<1 / 2$, there exists $c>0$ such that

$$
\frac{1}{\left|\mathrm{H}_{k}^{*}(N)\right|} \mid\left\{f \in \mathrm{H}_{k}^{*}(N) \mid \lambda_{f}(p) \text { has sign } \varepsilon_{p} \text { for } p \leqslant z, p \nmid N\right\} \mid \geqslant\left(\frac{1}{2}-\varepsilon\right)^{\pi(z)}
$$

for $z=c \sqrt{(\log k N)(\log \log k N)}$, provided $k N$ is large enough.

One may expect that the same result would be true for $z \leqslant c \log k N$ (note that

$$
\left(\frac{1}{2}\right)^{\pi(c \log k N)} \geqslant \exp \left(-c_{1} \frac{\log k N}{\log \log k N}\right)
$$

so this result would be quite close to the statistic upper-bound of Corollary 1, and would essentially be best possible, confirming that the signs of $\lambda_{f}(p)$ behave almost like independent (and unbiased) random variables in that range of $p$ ).

\footnotetext{
${ }^{2}$ The cases where $\nu \geqslant 2$ can be interpreted as similar statements for the $\nu$-th symmetric powers.
} 
Theorems 2 and 3 will be proved in Section 3, using the method in [13] and quantitative equidistribution statements for Hecke eigenvalues, respectively.

1.3. Recognition of modular forms by signs of Hecke eigenvalues. Here we consider whether it is true that a primitive form $f$ is determined uniquely by the sequence of signs of its Fourier coefficients $\lambda_{f}(p)$, where we recall that we interpret the sign of 0 in a relaxed way, so that 0 has the same sign as both positive and negative numbers.

The answer to this question is, indeed, yes, and in fact (in the non-CM case) an analogue of the strong multiplicity one theorem holds: not only can we exclude finitely many primes, or a set of primes of density zero, but even a set of sufficiently small positive density. Here, the density we use is the analytic density defined as follows: a set $E$ of primes has density $\kappa>0$ if and only if

$$
\sum_{p \in E} \frac{1}{p^{\sigma}} \sim \kappa \sum_{p} \frac{1}{p^{\sigma}} \sim-\kappa \log (\sigma-1) \quad(\sigma \rightarrow 1+) .
$$

We will prove:

Theorem 4. Let $k_{1}, k_{2} \geqslant 2$ be even integers, let $N_{1}, N_{2} \geqslant 1$ be integers and $f_{1} \in$ $\mathrm{H}_{k_{1}}^{*}\left(N_{1}\right), f_{2} \in \mathrm{H}_{k_{2}}^{*}\left(N_{2}\right)$.

(1) If the signs of $\lambda_{f_{1}}(p)$ and $\lambda_{f_{2}}(p)$ are the same for all $p$ except those in a set of analytic density 0 , then $f_{1}=f_{2}$.

(2) Assume that neither of $f_{1}$ and $f_{2}$ is of CM type, for instance assume that $N_{1}$ and $N_{2}$ are squarefree. Then, if $\lambda_{f_{1}}(p)$ and $\lambda_{f_{2}}(p)$ have same sign for every prime $p$, except those in a set $E$ of analytic density $\kappa$, with $\kappa \leqslant 1 / 32$, it follows that $f_{1}=f_{2}$.

Recall that a form $f \in \mathrm{H}_{k}^{*}(N)$ is of CM type if there exists a non-trivial primitive real Dirichlet character $\chi$ such that $\lambda_{f}(p)=\chi(p) \lambda_{f}(p)$ for all but finitely many primes $p$. In that case, $\lambda_{f}(p)=0$ for all $p$ such that $\chi(p)=-1$, and hence its signs coincide (in our relaxed sense) with those of any other modular form for a set of primes of density at least $1 / 2$.

Of course, Theorem 4 is also valid for the natural density, since the existence of the latter implies that of the analytic density, and that they are equal. As a corollary, we get of course:

Corollary 2. For any sequence of signs $\left(\varepsilon_{p}\right)$ indexed by primes, there is at most pair $(k, N)$ and one $f \in \mathrm{H}_{k}^{*}(N)$ such that $\lambda_{f}(p)$ has sign $\varepsilon_{p}$ for all primes.

Theorem 4 is proved in Section 4 . The argument is short and simple, but it depends crucially on a very deep result: Ramakrishnan's proof [18] the RankinSelberg convolution $L$-function is the $L$-function of some modular form on $G L(4)$.

1.4. Motivation, further remarks and problems. The main remark is that, underlying most of the problems we consider is the Sato-Tate conjecture, which we recall (see Mazur's survey [14]): provided $f$ is not of CM type (for instance, if $N$ is squarefree), one should have

$$
\lim _{x \rightarrow+\infty} \frac{1}{\pi(x)}\left|\left\{p \leqslant x \mid \theta_{f}(p) \in[\alpha, \beta]\right\}\right|=\int_{\alpha}^{\beta} \mathrm{d} \mu_{S T}
$$


for any $\alpha<\beta$, where $\mu_{S T}$ is the Sato-Tate measure

$$
\mu_{S T}=\frac{2}{\pi} \sin ^{2} \theta \mathrm{d} \theta
$$

on $[0, \pi]$. Since $\mu_{S T}([0, \pi / 2])=\mu_{S T}([\pi / 2, \pi])$, this indicates in particular that the signs of $\lambda_{f}(p)$ should be equitably shared between +1 and -1 . This suggests and motivates many of our results and techniques of proof.

There is much ongoing progress on the Sato-Tate conjecture; for $f \in \mathrm{H}_{k}^{*}(N)$, non-CM, a proof of the conjecture has been announced by Barnet-Lamb, Geraghty, Harris and Taylor [1, Th. B]. However, knowing its truth does not immediately simplify our arguments. Indeed, it would be most immediately relevant for parts of Section 4, but Theorem 4 is really concerned with the pair-Sato-Tate conjecture, or property, which would be the statement, for a pair $\left(f_{1}, f_{2}\right)$, that for any $a_{1}<b_{1}$, $a_{2}<b_{2}$ the set of primes

$$
\left\{p \mid \lambda_{f_{1}}(p) \in\left[a_{1}, b_{1}\right] \text { and } \lambda_{f_{2}}(p) \in\left[a_{2}, b_{2}\right]\right\}
$$

has density equal to $\mu_{S T}\left(\left[a_{1}, b_{1}\right]\right) \mu_{S T}\left(\left[a_{2}, b_{2}\right]\right)$ (in other words, the Fourier coefficients at primes are independently Sato-Tate distributed). This is expected to hold for any pair of non-CM modular forms, such that neither is a quadratic twist of the other (and in the case of elliptic curves, Mazur [14, Footnote 12] mentions that there is ongoing progress by Harris on this problem). If this holds, it will follow that the density of coincidences of signs is always $\leqslant \frac{1}{2}$, which is the probability under independent Sato-Tate measures that two "samples" are of the same sign.

Here is a natural question which is suggested by Theorem 4: estimate the size, as a function of the weight and conductor, of the smallest integer $n_{f_{1}, f_{2}}$ for which the sign of $\lambda_{f_{1}}(n)$ and $\lambda_{f_{2}}(n)$ are different. If we enlarge slightly our setting to allow $f_{2}$ to be an Eisenstein series such as the Eisenstein series of weight 4:

$$
E_{4}(z)=1+240 \sum_{n \geqslant 1}\left(\sum_{d \mid n} d^{3}\right) e(n z),
$$

where all Hecke eigenvalues are positive, then the question becomes (once more) that of finding the first negative Hecke eigenvalue for $f_{1}$, i.e., the problem considered in Theorem 1. Hence, we know that

$$
n_{f_{1}, E_{4}} \ll\left(k_{1}^{2} N_{1}\right)^{10 / 21}
$$

where the implied constant is absolute, but it would be interesting to obtain a more general version, in particular a uniform one with respect to both $f_{1}$ and $f_{2}$.

At least our statistic result (Theorem 2) generalizes immediately if one of the forms is fixed: taking $\mathscr{P}$ to be the set of all primes, $\nu=1$ and $\varepsilon_{p}=\operatorname{sign} \lambda_{f_{2}}(p)$ if $\lambda_{f_{2}}(p) \neq 0$, and 1 otherwise, we get immediately the following corollary:

Corollary 3. Let $k_{1}, k_{2} \geqslant 2$ be even integers and $N_{1}, N_{2} \geqslant 1$ squarefree. For any fixed $f_{2} \in \mathrm{H}_{k_{2}}^{*}\left(N_{2}\right)$, there is an absolute positive constant $c$ such that

$$
n_{f_{1}, f_{2}} \ll f_{2} \log \left(k_{1} N_{1}\right)
$$

for all $f \in \mathrm{H}_{k_{1}}^{*}\left(N_{1}\right)$ except for those in an exceptional set with

$$
\ll k_{1} N_{1} \exp \left(-c \frac{\log k_{1} N_{1}}{\log \log k_{1} N_{1}}\right)
$$


elements, where the implied constants depend only on $f_{2}$.

\section{Proof of Theorem 1}

Although some ideas are related to those of Iwaniec, Kohnen and Sengupta, the proof of Theorem 1 is somewhat easier.

Thus let $f \in H_{k}^{*}(N)$, and let $y>0$ be such that $\lambda_{f}(n) \geqslant 0$ for $n \leqslant y$ and $(n, N)=1$. The idea to estimate $y$ is to compare upper and lower bounds for the sum

$$
S(f, x)=\sum_{\substack{n \leqslant x \\(n, N)=1}}^{b} \lambda_{f}(n)
$$

where $\sum^{b}$ restricts a sum to squarefree integers. An upper bound is easily achieved: using the convexity bound for Hecke $L$-functions and the Perron formula, we obtain

$$
S(f, x) \ll_{\varepsilon}\left(k^{2} N\right)^{1 / 4+\varepsilon} x^{1 / 2+\varepsilon} \quad(x \geqslant 1) .
$$

This estimate is independent of any information on $y$. We note that, more generally, if we have

$$
L(f, 1 / 2+i t) \ll\left(k^{2} N(1+|t|)^{2}\right)^{\eta},
$$

for $t \in \mathbb{R}$, where $\eta>0$, then we get (2.1) for $x \geqslant Q^{2 \eta+\varepsilon}$ (the recent work of Michel and Venkatesh [15] provides such a uniform result for some - very small $-\eta<1 / 4$, and the convexity bound states that any $\eta>1 / 4$ is suitable).

We now proceed to establish a lower bound for $S(f, x)$ by using the assumption. For primes $p \leqslant y$ with $p \nmid N$, we thus have $\lambda_{f}(p) \geqslant 0$; furthermore, if $p \leqslant \sqrt{y}$ and $p \nmid N$, we have the better bound $\lambda_{f}(p) \geqslant 1$ because

$$
\lambda_{f}(p)^{2}=1+\lambda_{f}\left(p^{2}\right) \geqslant 1
$$

by the Hecke relation.

We now introduce an auxiliary multiplicative function $h=h_{y}$ defined by

$$
h_{y}(p)= \begin{cases}-2 & \text { if } p>y \text { and } p \nmid N \\ 0 & \text { if } \sqrt{y}<p \leqslant y \text { or } p \mid N \\ 1 & \text { if } p \leqslant \sqrt{y} \text { and } p \nmid N\end{cases}
$$

and $h_{y}\left(p^{\nu}\right)=0$ for $\nu \geqslant 2$.

We shall show in a moment the following lemma:

Lemma 2.1. For any $y>0$, define $h_{y}$ as above. Then, for any $\varepsilon>0$, we have

$$
\sum_{n \leqslant y^{u}} h_{y}(n)=\zeta_{M}(2)^{-1} \frac{\varphi(N)}{N} y^{u}(\rho(2 u)-2 \log u)\left\{1+O\left(\frac{\left(\log _{2} y\right)^{2}}{\log y}\right)\right\}
$$

uniformly for

$$
1 \leqslant u \leqslant \frac{3}{2} \quad \text { and } \quad y \geqslant N^{1 / 3}
$$

where

$$
\zeta_{N}(2)=\prod_{p \nmid N}\left(1-p^{-2}\right)^{-1}
$$


and $\rho(u)$ is the Dickman function, defined as the unique continous solution of the difference-differential equation

$$
u \rho^{\prime}(u)+\rho(u-1)=0 \quad(u>1), \quad \rho(u)=1 \quad(0<u \leqslant 1) .
$$

In particular $\rho(2 u)-2 \log u>0$ for all $u<\kappa$ where $\kappa$ is the solution to $\rho(2 \kappa)=$ $2 \log \kappa$. We have $\kappa>\frac{10}{9}$.

The point of introducing this auxiliary function is that, with notation as before, we have the lower bound

$$
S\left(f, y^{u}\right) \geqslant \sum_{n \leqslant y^{u}} h_{y}(n)
$$

for all $u<\kappa$, provided $y$ is large enough, e.g., $y \geqslant N^{1 / 3}$ with $N$ large enough, which we can obviously assume in proving Theorem 4 .

Indeed, let $g_{y}$ be the multiplicative function defined by the Dirichlet convolution identity

$$
\lambda_{f}=g_{y} * h_{y}
$$

then $g_{y}(n) \geqslant 0$ for all squarefree integers $n \geqslant 1$ such that $(n, N)=1$, since

$$
g_{y}(p)=\lambda_{f}(p)-h_{y}(p) \geqslant 0
$$

for all $p \nmid N$ (the case $p \geqslant y$ following from Deligne's inequality). We have trivially

$$
\sum_{n \leqslant z} h_{y}(n) \geqslant 0
$$

if $z \leqslant y$ since each term is non-negative in that range, and additionally

$$
\sum_{n \leqslant y^{u}} h_{y}(n) \geqslant 0
$$

for $u<\kappa$ if $y$ is large enough, by Lemma 2.1 .

Hence

$$
S\left(f, y^{u}\right)=\sum_{\substack{n \leqslant y^{u} \\(n, N)=1}}^{b} \lambda_{f}(n)=\sum_{d \leqslant y^{u}}^{b} g_{y}(d) \sum_{\ell \leqslant y^{u} / d}^{b} h_{y}(\ell) \geqslant \sum_{\ell \leqslant y^{u}}^{b} h_{y}(\ell)
$$

since every term in the sum over $d$ is non-negative and $g_{y}(1)=1$.

We now deduce from Lemma 2.1

$$
S\left(f, y^{u}\right) \geqslant \sum_{n \leqslant y^{u}} h_{y}(n) \gg \frac{y^{u}}{\log \log N},
$$

for $u<\kappa$. Then, a comparison with (2.1) gives the estimate

$$
y \leqslant\left(k^{2} N\right)^{1 /(2 \kappa)+o(1)} .
$$

Quoting the lower bound for $\kappa$ from Lemma 2.1, we are done.

Proof of Lemma 2.1. According to the definition of $h_{y}$, we have

$$
\sum_{n \leqslant y^{u}} h_{y}(n)=\sum_{\substack{n \leqslant y^{u} \\ P(n) \leqslant \sqrt{y}}}^{b} 1-2 \sum_{\substack{y<p \leqslant y^{u} \\ p \nmid N}} \sum_{m \leqslant y^{u} / p}^{b} 1
$$


for all $u$ and $y$ satisfying (2.3), where we use the convention here that $\sum^{b}$ also restricts the sum to $(n, N)=1$ to simplify notation.

The second term contributes

$$
-\frac{2}{\zeta_{N}(2)} \frac{\varphi(N)}{N} y^{u}(\log u)\left\{1+O\left(\frac{\left(\log _{2} y\right)^{2}}{\log y}\right)\right\}
$$

by standard estimates (the leading constant arises of course because

$$
\sum_{\substack{n \leqslant y^{u} \\(n, N)=1}}^{b} 1 \sim \frac{1}{\zeta_{N}(2)} \frac{\varphi(N)}{N} y^{u}
$$

uniformly in our range).

For the first term, if it were not for this condition and the requirement that $n$ be squarefree, the lemma would then follow immediately from the well-known property

$$
\sum_{\substack{n \leqslant y^{u} \\ P(n) \leqslant \sqrt{y}}} 1=\rho(2 u) y^{u}\left\{1+O\left(\frac{1}{\log y}\right)\right\},
$$

of the Dickman function. However, because our uniformity requirements are quite modest, it is fairly simple to deduce the stated inequality from this result using Möbius inversion to detect the coprimality and squarefree condition (for very general bounds of this type, see [26, Th. 2.1], though our requirements are much weaker).

A numerical computation using MAPLE leads to $\kappa>\frac{10}{9}$.

Remark 1. To estimate $\kappa$, one can also use the lower bound

$$
\rho(2 u)=1-\log (2 u)+\int_{2}^{2 u} \frac{\log (t-1)}{t} \mathrm{~d} t \geqslant 1-\log (2 u) \quad\left(1 \leqslant u \leqslant \frac{3}{2}\right),
$$

which shows that $\rho(2 u)-2 \log u \geqslant 1-\log 2-3 \log u$, and

$$
\kappa \geqslant(e / 2)^{1 / 3}>\frac{11}{10},
$$

which leads to the exponent $\frac{5}{11}=0.4545 \ldots$ in Theorem 1 .

Remark 2. This result is not the limit of the method employed. Precisely, in addition to obtained $\lambda_{f}(p) \geqslant 1$ for $p<\sqrt{y},(p, N)=1$, we can exploit higher powers: write $\lambda_{f}(p)=2 \cos \theta_{f}(p)$ with $\theta_{f}(p) \in[0, \pi]$. Then if $m \geqslant 1$ is an integer, we have for $1 \leqslant j \leqslant m$ and $y^{1 /(m+1)} \leqslant p<y^{1 / m}$ that

$$
0 \leqslant \lambda_{f}\left(p^{j}\right)=\frac{\sin \left((j+1) \theta_{f}(p)\right)}{\sin \theta_{f}(p)}
$$

(if $p \nmid N)$. This implies that $\theta_{f}(p) \leqslant \pi /(m+1)$, and hence

$$
\lambda_{f}(p) \geqslant 2 \cos \frac{\pi}{m+1},
$$

for $p \nmid N$ with $y^{1 /(m+1)} \leqslant p<y^{1 / m}$. This can be exploited by boundind $S(f, y)$ from below using a new auxiliary function $h$ supported on squarefree numbers coprime to $N$ with

$$
h(p)=\alpha\left(\frac{\log p}{\log y}\right)
$$


where $\alpha(u)=-2$ for $u \geqslant 1, \alpha(0)=2$ and $\alpha(u)=2 \cos (\pi /(m+1))$ if $1 /(m+1) \leqslant$ $u<1 / m$. One can show an asymptotic of the type

$$
\sum_{n \leqslant y^{u}} h(n) \sim C \beta(u) y^{u}\left(\log y^{u}\right)
$$

(assuming $N=1$ for simplicity) for some constant $C>0$, where the function $\beta$ can be described by the following inclusion-exclusion formula:

$$
u \beta(u)=u+\sum_{j \geqslant 1} \frac{(-1)^{j}}{j !} I_{j}(u)
$$

with

$$
I_{j}(u)=\int_{\Delta_{j}}\left(u-t_{1}-\cdots-t_{j}\right) \prod_{i=1}^{j}\left(2-\alpha\left(t_{j}\right)\right) \frac{d t_{1} \cdots d t_{j}}{t_{1} \cdots t_{j}}
$$

integration ranging over the set

$$
\Delta_{j}=\left\{( t _ { 1 } , \ldots , t _ { j } ) \in \left[0,+\infty\left[^{j} \mid t_{1}+\cdots+t_{j} \leqslant u\right\} .\right.\right.
$$

This function is also a solution of the integral equation

$$
u^{2} \beta(u)=\int_{0}^{u} t \beta(t) \alpha(u-t) d t
$$

(see [5] for related investigations of a class of integral equations of this type).

To improve Theorem 1, one needs to find (a close approximation to) the first positive zero of $\beta$. We have not found a nice way to compute $\beta$ numerically, but this would be quite an interesting problem, and its solution is likely to lead to significant improvements in the result. We hope to come back to this in the future.

\section{Statistical Results}

Our goal is now to prove Theorems 2 and 3. For the first, the main tool is the following type of large sieve inequality.

Lemma 3.1 ([13], Theorem 1). Let $\nu \geqslant 1$ be a fixed integer and let $\left\{b_{p}\right\}_{p}$ be a sequence of real numbers indexed by prime numbers such that $\left|b_{p}\right| \leqslant B$ for some constant $B$ and for all primes $p$. Then we have

$$
\sum_{f \in \mathrm{H}_{k}^{*}(N)}\left|\sum_{\substack{P<p \leqslant Q \\ p \nmid N}} b_{p} \frac{\lambda_{f}\left(p^{\nu}\right)}{p}\right|^{2 j} \ll_{\nu} k \varphi(N)\left(\frac{96 B^{2}(\nu+1)^{2} j}{P \log P}\right)^{j}+(k N)^{10 / 11}\left(\frac{10 B Q^{\nu / 10}}{\log P}\right)^{2 j}
$$

uniformly for

$$
B>0, \quad j \geqslant 1, \quad 2 \mid k, \quad 2 \leqslant P<Q \leqslant 2 P, \quad N \geqslant 1 \quad \text { (squarefree). }
$$

The implied constant depends on $\nu$ only.

Proof of Theorem 2. The basic idea is that for all forms $f$ with coefficients $\lambda_{f}\left(p^{\nu}\right)$ of the same sign $\varepsilon_{p}$, the sums

$$
\sum_{\substack{P<p \leqslant 2 P \\ p \in \mathscr{P}}} \frac{\varepsilon_{p} \lambda_{f}\left(p^{\nu}\right)}{p}
$$


exhibit no cancellation due to variation of signs. The large sieve implies this is very unlikely to happen, except if the $\lambda_{f}\left(p^{\nu}\right)$ are very small in absolute value. The Hecke relations are used to control this other possibility by relating it to $\lambda_{f}\left(p^{2 \nu}\right)$ being large which can not happen too often either. ${ }^{3}$

For the details, we first denote

$$
\mathscr{P}_{N}:=\{p \in \mathscr{P} \mid p \nmid N\},
$$

and define

$$
\begin{aligned}
\mathscr{E}_{k}^{*}(N, P ; \mathscr{P}) & :=\left\{f \in \mathrm{H}_{k}^{*}(N) \mid \varepsilon_{p} \lambda_{f}\left(p^{\nu}\right)>0 \text { for } p \in \mathscr{P}_{N} \cap(P, 2 P]\right\} \\
\mathscr{E}_{k}^{\nu^{\prime}}(N, P ; \mathscr{P}) & :=\left\{f \in \mathrm{H}_{k}^{*}(N)|| \sum_{\substack{P<p \leqslant 2 P \\
p \in \mathscr{P}_{N}}} \frac{\lambda_{f}\left(p^{2 \nu^{\prime}}\right)}{p} \mid \geqslant \frac{\delta}{2 \nu \log P}\right\} \quad\left(1 \leqslant \nu^{\prime} \leqslant \nu\right) .
\end{aligned}
$$

To prove Theorem 2, clearly we only need to show that there are two positive constants $C=C(\nu, \mathscr{P})$ and $c=c(\nu, \mathscr{P})$ such that

$$
\left|\mathscr{E}_{k}^{*}(N, P ; \mathscr{P})\right| \ll_{\nu, \mathscr{P}} k N \exp \left(-c \frac{\log k N}{\log \log k N}\right)
$$

uniformly for

$$
2 \mid k, \quad N \quad \text { (squarefree), } \quad k N \geqslant X_{0}, \quad C \log (k N) \leqslant P \leqslant(\log (k N))^{10}
$$

for some sufficiently large number $X_{0}=X_{0}(\nu, \mathscr{P})$.

The definition of $\mathscr{E}_{k}^{*}(N, P ; \mathscr{P})$ and Deligne's inequality allow us to write

$$
\begin{aligned}
\sum_{f \in \mathscr{E}_{k}^{*}(N, P ; \mathscr{P})}\left|\sum_{\substack{P<p \leqslant 2 P \\
p \in \mathscr{P}_{N}}} \frac{\lambda_{f}\left(p^{\nu}\right)^{2}}{p}\right|^{2 j} & \leqslant \sum_{f \in \mathscr{E}_{k}^{*}(N, P ; \mathscr{P})}\left|\sum_{\substack{P<p \leqslant 2 P \\
p \in \mathscr{P}_{N}}}(\nu+1) \varepsilon_{p} \frac{\lambda_{f}\left(p^{\nu}\right)}{p}\right|^{2 j} \\
& \leqslant \sum_{f \in \mathrm{H}_{k}^{*}(N)}\left|\sum_{\substack{P<p \leqslant 2 P \\
p \in \mathscr{P}_{N}}}(\nu+1) \varepsilon_{p} \frac{\lambda_{f}\left(p^{\nu}\right)}{p}\right|^{2 j}
\end{aligned}
$$

Choosing

$$
b_{p}= \begin{cases}(\nu+1) \varepsilon_{p} & \text { if } p \in \mathscr{P}, \\ 0 & \text { otherwise }\end{cases}
$$

in Lemma 3.1, we find that

$$
\begin{aligned}
\sum_{f \in \mathscr{E}_{k}^{*}(N, P ; \mathscr{P})}\left|\sum_{\substack{P<p \leqslant 2 P \\
p \in \mathscr{P}_{N}}} \frac{\lambda_{f}\left(p^{\nu}\right)^{2}}{p}\right|^{2 j} & \leqslant \sum_{f \in \mathrm{H}_{k}^{*}(N)}\left|\sum_{\substack{P<p \leqslant 2 P \\
p \nmid N}} b_{p} \frac{\lambda_{f}\left(p^{\nu}\right)}{p}\right|^{2 j} \\
& \ll k N\left(\frac{96(\nu+1)^{4} j}{P \log P}\right)^{j}+(k N)^{10 / 11} P^{\nu j / 2} .
\end{aligned}
$$

\footnotetext{
${ }^{3}$ Variants of this well-known trick have been used in a number of other contexts, as in [3], but note that the large sieve inequality proved there would not work for this problem, due to the lack of multiplicative stability of the sign conditions (it would also be much less efficient).
} 
In view of the Hecke relation (1.1), the left-hand side of (3.2) is

$$
\begin{aligned}
& \geqslant \sum_{f \in \mathscr{E}_{k}^{*}(N, P ; \mathscr{P}) \backslash\left(\cup_{\nu^{\prime}=1}^{\nu^{\prime}=1_{k}} \mathscr{E}_{k}^{\nu^{\prime}}(N, P ; \mathscr{P})\right)}\left(\sum_{\substack{P<p \leqslant 2 P \\
p \in \mathscr{P}_{N}}} \frac{1}{p}-\sum_{1 \leqslant \nu^{\prime} \leqslant \nu}\left|\sum_{\substack{P<p \leqslant 2 P \\
p \in \mathscr{P}_{N}}} \frac{\lambda_{f}\left(p^{2 \nu^{\prime}}\right)}{p}\right|\right)^{2 j} \\
& \geqslant \sum_{f \in \mathscr{E}_{k}^{*}(N, P ; \mathscr{P}) \backslash\left(\cup_{\nu^{\prime}=1}^{\nu} \mathscr{E}_{k}^{\nu^{\prime}}(N, P ; \mathscr{P})\right)}\left(\sum_{\substack{P<p \leqslant 2 P \\
p \in \mathscr{P}_{N}}} \frac{1}{p}-\frac{\delta}{2 \log P}\right)^{2 j} .
\end{aligned}
$$

Let $\omega(n)$ be the number of distinct prime factors of $n$. Using the hypothesis on $\mathscr{P}$ and the classical inequality

we infer that

$$
\omega(n) \leqslant\{1+o(1)\} \frac{\log n}{\log \log n},
$$

$$
\begin{aligned}
\sum_{\substack{P<p \leqslant 2 P \\
p \in \mathscr{P}_{N}}} \frac{1}{p}-\frac{\delta}{2 \log P} & \geqslant \sum_{\substack{P<p \leqslant 2 P \\
p \in \mathscr{P}}} \frac{1}{p}-\sum_{\substack{P<p \leqslant 2 P \\
p \mid N}} \frac{1}{p}-\frac{\delta}{2 \log P} \\
& \geqslant \frac{\delta}{2 \log P}-\frac{\omega(N)}{P} \geqslant \frac{\delta / 2-2 / C}{\log P} \geqslant \frac{\delta}{6 \log P},
\end{aligned}
$$

provided $C \geqslant 6 / \delta$. Combining this with (3.2), we infer that

$\left|\mathscr{E}_{k}^{*}(N, P ; \mathscr{P}) \backslash\left(\cup_{\nu^{\prime}=1}^{\nu} \mathscr{E}_{k}^{\nu^{\prime}}(N, P ; \mathscr{P})\right)\right| \ll k N\left(\frac{3456(\nu+1)^{4} j \log P}{\delta^{2} P}\right)^{j}+(k N)^{10 / 11} P^{j}$

Now we bound the size of the sets $\mathscr{E}_{k}^{\nu^{\prime}}(N, P ; \mathscr{P})$ to finish the proof. Taking

$$
B=1, \quad \nu=2 \nu^{\prime}, \quad Q=2 P \quad \text { and } \quad b_{p}= \begin{cases}1 & \text { if } p \in \mathscr{P} \\ 0 & \text { otherwise }\end{cases}
$$

in Lemma 3.1, we get

$$
\begin{aligned}
\left(\frac{\delta}{2 \log P}\right)^{2 j}\left|\mathscr{E}_{k}^{\nu^{\prime}}(N, P ; \mathscr{P})\right| & \leqslant \sum_{f \in \mathrm{H}_{k}^{*}(N)}\left|\sum_{\substack{P<p \leqslant 2 P \\
p \nmid N}} b_{p} \frac{\lambda_{f}\left(p^{2 \nu^{\prime}}\right)}{p}\right|^{2 j} \\
& \ll k N\left(\frac{96\left(2 \nu^{\prime}+1\right)^{2} j}{P \log P}\right)^{j}+(k N)^{10 / 11}\left(\frac{10(2 P)^{\nu^{\prime} / 5}}{\log P}\right)^{2 j} .
\end{aligned}
$$

Hence,

$$
\left|\mathscr{E}_{k}^{\nu^{\prime}}(N, P ; \mathscr{P})\right| \ll k N\left(\frac{3456 \nu^{4} j \log P}{\delta^{2} P}\right)^{j}+(k N)^{10 / 11} P^{\nu j} \quad\left(1 \leqslant \nu^{\prime} \leqslant \nu\right)
$$

provided $P \geqslant 2(20 \nu / \delta)^{10 /(3 \nu)}$.

Combining this with (3.3), we finally obtain

$$
\left|\mathscr{E}_{k}^{*}(N, P ; \mathscr{P})\right| \ll k N\left(\frac{3456(\nu+1)^{4} j \log P}{\delta^{2} P}\right)^{j}+(k N)^{10 / 11} P^{\nu j}
$$

uniformly for

$2 \mid k, \quad N \quad$ (squarefree) $, \quad C \log (k N) \leqslant P \leqslant(\log (k N))^{10}, \quad j \geqslant 1$. 
Now, take

$$
j=\left[\delta^{*} \frac{\log (k N)}{\log P}\right]
$$

where $\delta^{*}=\delta^{2} /(10(\nu+1))^{4}$. We can ensure $j>1$ once $X_{0}$ is chosen to be suitably large. A simple computation gives that

$$
\left(\frac{3456(\nu+1)^{4} j \log P}{\delta^{2} P}\right)^{j} \ll \exp \left(-c \frac{\log k N}{\log \log k N}\right)
$$

for some positive constant $c=c(\nu, \mathscr{P})$ and $P^{\nu j} \ll(k N)^{1 / 1000}$, provided $X_{0}$ is large enough. Inserting them into (3.4), we get (3.1) and complete the proof.

We now come to the lower bound of Theorem 3. Our basic tool here is an equidistribution theorem for Hecke eigenvalues which is of some independent interest: it shows (quantitatively) that, after suitable average over $\mathrm{H}_{k}^{*}(N)$, the Hecke eigenvalues corresponding to the first primes are independently Sato-Tate distributed (thus, it is related to the earlier work of Sarnak [20] for Maass forms and Serre [21] and Royer [19] for holomorphic forms).

First, we recall the definition (1.4) of the angle $\theta_{f}(p) \in[0, \pi]$ associated to any $f \in \mathrm{H}_{k}^{*}(N)$ and prime $p \nmid N$. We also recall that the Chebychev functions $X_{n}$, $n \geqslant 0$, defined by

$$
X_{n}(\theta)=\frac{\sin ((n+1) \theta)}{\sin \theta}
$$

for $\theta \in[0, \pi]$, form an orthonormal basis of $L^{2}\left([0, \pi], \mu_{S T}\right)$. Hence, for any $\omega \geqslant 1$, the functions of the type

$$
\left(\theta_{1}, \ldots, \theta_{\omega}\right) \mapsto \prod_{1 \leqslant j \leqslant \omega} X_{n_{j}}\left(\theta_{j}\right)
$$

for $n_{j} \geqslant 0$, form an orthonormal basis of $L^{2}\left([0, \pi]^{\omega}, \mu_{S T}^{\otimes \omega}\right)$.

Proposition 1. Let $N$ be a squarefree number, $k \geqslant 2$ an even integer, $s \geqslant 1$ an integer and $z \geqslant 2$ a real number. For any prime $p \leqslant z$ coprime with $N$, let

$$
Y_{p}(\theta)=\sum_{j=0}^{s} \hat{y}_{p}(j) X_{j}(\theta)
$$

be a "polynomial" of degree $\leqslant s$ expressed in the basis of Chebychev functions on $[0, \pi]$. Then we have

$$
\sum_{f \in \mathrm{H}_{k}^{*}(N)} \omega_{f} \prod_{\substack{p \leqslant z \\(p, N)=1}} Y_{p}\left(\theta_{f}(p)\right)=\prod_{\substack{p \leqslant z \\(p, N)=1}} \hat{y}_{p}(0)+O\left(C^{\pi(z)} D^{s z}(\tau(N) \log 2 N)^{2}\left(N k^{5 / 6}\right)^{-1}\right)
$$

where

$$
\begin{gathered}
\omega_{f}=\frac{\Gamma(k-1)}{(4 \pi)^{k-1}\langle f, f\rangle} \frac{N}{\varphi(N)}, \quad\langle f, f\rangle \text { the Petersson norm of } f, \\
C=\max _{p, j}\left|\hat{y}_{p}(j)\right|,
\end{gathered}
$$

and $D \geqslant 1$ and the implied constant are absolute. 
By linearity, clearly, we get an analogue result for

$$
\sum_{f \in \mathrm{H}_{k}^{*}(N)} \omega_{f} \varphi\left(\left(\theta_{p}\right)_{p \leqslant z}\right), \quad \varphi=\sum_{j} \varphi_{j},
$$

where each $\varphi_{j}$ is a function which is a product of polynomials as in the statement. Proof. Using the fact that for any $n_{p} \geqslant 0$, we have

$$
\prod_{\substack{p \leqslant z \\(p, N)=1}} X_{n_{p}}\left(\theta_{f}(p)\right)=\lambda_{f}\left(\prod_{\substack{p \leqslant z \\(p, N)=1}} p^{n_{p}}\right)
$$

(which is another form of the Hecke multiplicativity), we expand the product and get

$$
\prod_{\substack{p \leqslant z \\(p, N)=1}} Y_{p}\left(\theta_{f}(p)\right)=\sum_{d \mid P_{N}(z)^{s}}\left(\prod_{p \mid P_{N}(z)} \hat{y}_{p}\left(v_{p}(d)\right)\right) \lambda_{f}(d)
$$

where $v_{p}(d)$ is the $p$-adic valuation of an integer and $P_{N}(z)$ is the product of the primes $p \leqslant z, p \nmid N$.

We now sum over $f$ and appeal to the following Petersson formula for primitive forms:

$$
\sum_{f \in \mathrm{H}_{k}^{*}(N)} \omega_{f} \lambda_{f}(m)=\delta(m, 1)+O\left(m^{1 / 4} \tau(N)^{2}(\log 2 m N)^{2}\left(N k^{5 / 6}\right)^{-1}\right),
$$

for all $m \geqslant 1$ coprime with $N$ (this is a simplified version of that in [8, Cor. 2.10]; note our slightly different definition of $\omega_{f}$, which explains the absence of $\varphi(N) / N$ on the right-hand side); the result then follows easily from simple estimates for the sum over $d$ of the remainder terms.

We now deduce Theorem 3 from this, assuming $\varepsilon_{p}=1$ for all $p$ (handling the other choices of signs being merely a matter of complicating the notation).

To simplify notation, we write $P=P_{N}(z)$ the product of primes $\leqslant z$ coprime with $N$, and $\omega$ the number of such primes.

First, if we wanted only to have $\lambda_{f}(p) \geqslant 0$ for a fixed (finite) set of primes (i.e., for $z$ fixed), we would be immediately done: Proposition 1 shows ${ }^{4}$ that the $\left(\theta_{f}(p)\right)_{p \mid P}$ become equidistributed as $k N \rightarrow+\infty$ with respect to the product SatoTate measure, if we weigh modular forms with $\omega_{f}$, and hence

$$
\sum_{\substack{f \in \mathrm{H}_{k}^{*}(N) \\ p \mid P \Rightarrow \lambda_{f}(p) \geqslant 0}} \omega_{f} \rightarrow \mu_{S T}([0, \pi / 2])^{\omega}=\left(\frac{1}{2}\right)^{\omega}
$$

which is of the desired type, except for the presence of the weight. ${ }^{5}$ However, we want to have

$$
\lambda_{f}(p) \geqslant 0 \text { for } p \leqslant z,(p, N)=1,
$$

\footnotetext{
${ }^{4}$ For Maass forms, this is essentially one of the early results of Sarnak [20].

${ }^{5}$ Using the trace formula instead of the Petersson formula (as in [19]), the unweighted analogue of Proposition 1 holds with a product of local Plancherel measures, but each still gives measure $1 / 2$ to the two signs.
} 
where $z$ grows with $k N$, and this involves quantitative lower bounds for approximation in large dimension, which requires more care. We use a result of Barton, Montgomery and Vaaler [2] for this purpose; although it is optimized for uniform distribution modulo 1 instead of the Sato-Tate context, but it is not difficult to adapt it here and this gives a quick and clean argument. ${ }^{6}$

Precisely, we consider $[0, \pi]^{\omega}$, with the product Sato-Tate measure, and we will write $\theta=\left(\theta_{p}\right)$ for the elements of this set; we also consider $[0,1]^{\omega}$ and we write $x=\left(x_{p}\right)$ for elements there.

For any positive odd integer $L$, we get from [2, Th. 7] two explicit trigonometric polynomials ${ }^{7}$ on $[0,1]^{\omega}$, denoted $A_{L}(x), B_{L}(x)$, such that

$$
A_{L}(\theta / \pi)-B_{L}(\theta / \pi) \leqslant \prod_{\substack{p \leqslant z \\(p, N)=1}} \chi\left(\theta_{p}\right)
$$

for all $\theta=\left(\theta_{p}\right) \in[0, \pi]^{\omega}$, where $\chi\left(\theta_{p}\right)$ is the characteristic function of $[0, \pi / 2] \subset[0, \pi]$ (precisely, we consider the functions denoted $A(x), B(x)$ in [2], with parameters $N=\omega$ and $u_{n}=0, v_{n}=1 / 2$ for all $n \leqslant \omega$; since $\left(v_{n}-u_{n}\right)(L+1)=(L+1) / 2$ is a positive integer, we are in the situation $\Phi_{u, v} \in \mathcal{B}_{N}(L)$ of loc. cit.).

Thus we have the lower bound

$$
\sum_{\substack{f \in \mathrm{H}_{k}^{*}(N) \\ \lambda_{f}(p) \geqslant 0 \text { for } p \mid P}} \omega_{f} \geqslant \sum_{f \in \mathrm{H}_{k}^{*}(N)} \omega_{f}\left(A_{L}\left(\theta_{f} / \pi\right)-B_{L}\left(\theta_{f} / \pi\right)\right),
$$

where $\theta_{f}=\left(\theta_{f}(p)\right)_{p}$.

Moreover, as we will explain below, $A_{L}(\theta / \pi)$ is a product of polynomials over each variable, and $B_{L}(\theta / \pi)$ is a sum of $\omega$ such products, and we can now apply Proposition 1 (and the remark following it) to the terms on the right-hand side. More precisely, we claim that the following lemma holds:

Lemma 3.2. With notation as above, we have:

(1) For any $\varepsilon \in(0,1 / 2)$, there exists constants $L_{0} \geqslant 1$, and $c>0$, such that the contribution $\Delta$ of the constant terms of the Chebychev expansions of $A_{L}(\theta / \pi)$ and $B_{L}(\theta / \pi)$ satisfies

$$
\Delta \geqslant\left(\frac{1}{2}-\varepsilon\right)^{\pi(z)}
$$

if $L$ is the smallest odd integer $\geqslant c \pi(z)$ and if $L \geqslant L_{0}$.

(2) All the coefficients in the expansion in terms of Chebychev functions of the factors in $A_{L}(\theta / \pi)$ or in the terms of $B_{L}(\theta / \pi)$ are bounded by 1 .

(3) The degrees, in terms of Chebychev functions, of the factors of $A_{L}(\theta / \pi)$ and of the terms of $B_{L}(\theta / \pi)$, are $\leqslant 2 L$.

Using this lemma, fixing $\varepsilon \in(0,1 / 2)$ and taking $L$ as in Part (1) (we can obviously assume $L \geqslant L_{0}$, since otherwise $z$ is bounded) we derive from Proposition 1 that

$$
\sum_{f \in \mathrm{H}_{k}^{*}(N)} \omega_{f}\left(A_{L}\left(\theta_{f} / \pi\right)-B_{L}\left(\theta_{f} / \pi\right)\right)=\Delta+O\left(D^{z \pi(z)}(\tau(N) \log 2 N)^{2}\left(N k^{5 / 6}\right)^{-1}\right)
$$

\footnotetext{
${ }^{6}$ This result was also used recently by Y. Lamzouri [11, $\left.\S 7\right]$, in a somewhat related context.

${ }^{7}$ Meaning, standard trigonometric polynomials of the type $\sum_{\ell} \alpha_{\ell} e(\ell \cdot x)$.
} 
for some absolute constants $D$, with $\Delta \geqslant(1 / 2-\varepsilon)^{\pi(z)}$. This is then $\gg(1 / 2-\varepsilon)^{\pi(z)}$, provided

$$
D^{z \pi(z)}(\tau(N) \log 2 N)^{2}\left(N k^{5 / 6}\right)^{-1} \ll\left(\frac{1}{2}-\varepsilon\right)^{\pi(z)}
$$

This condition is satisfied for

$$
z \leqslant c \sqrt{(\log k N)(\log \log k N)}
$$

where $c>0$ is an absolute constant, and this gives Theorem 3 when counting with the weight $\omega_{f}$. But, using well-known bounds for $\langle f, f\rangle$, we have

$$
\omega_{f} \ll k N(\log k N)(\log \log 6 N) \ll k N(\log k N)^{2},
$$

with an absolute implied constant. Hence, for $z=c \sqrt{(\log k N)(\log \log k N)}$, we get

$$
\begin{aligned}
\frac{1}{\left|\mathrm{H}_{k}^{*}(N)\right|} \mid\left\{f \in \mathrm{H}_{k}^{*}(N) \mid \lambda_{f}(p) \geqslant 0 \text { for } p \leqslant z, p \nmid N\right\} \mid & \gg \frac{1}{(\log k N)^{2}}\left(\frac{1}{2}-\varepsilon\right)^{\pi(z)} \\
& \gg\left(\frac{1}{2}-2 \varepsilon\right)^{\pi(z)}
\end{aligned}
$$

if $k N$ is large enough, and so we obtain Theorem 3 as stated.

Proof of Lemma 3.2. We must now refer to the specific construction in [2]. We start with $A_{L}(x)$ : we have the product formula

$$
A_{L}(x)=\prod_{p \mid P} \alpha_{L}\left(x_{p}\right),
$$

where $\alpha_{L}$ is a trigonometric polynomial in one variable of degree $\leqslant L$, i.e., of the type

$$
\alpha_{L}(x)=\sum_{|\ell| \leqslant L} \hat{\alpha}_{L}(\ell) e(\ell x),
$$

with $\hat{\alpha}_{L}(0)=1 / 2($ see $[2,(2.2)$, Lemma $5,(2.17)])$. In particular, the constant term (in the Chebychev expansion) for $A_{L}(\theta / \pi)$ is given by

$$
\left(\int_{0}^{\pi} \alpha_{L}(\theta / \pi) \mathrm{d} \mu_{S T}\right)^{\omega}
$$

and we will bound it below. For the moment, we observe further that, from $[2$, Lemma 5], we know that $0 \leqslant \alpha_{L}(x) \leqslant 1$ for all $x \in[0,1]$, and so we can simply bound all the coefficients in the Chebychev expansion, using the Cauchy-Schwarz inequality and orthonormality:

$$
\begin{aligned}
\left|\int_{0}^{\pi} \alpha_{L}(\theta / \pi) X_{n}(\theta) \mathrm{d} \mu_{S T}\right|^{2} & \leqslant \int_{0}^{\pi}\left|\alpha_{L}(\theta / \pi)\right|^{2} \mathrm{~d} \mu_{S T} \times \int_{0}^{\pi}\left|X_{n}(\theta)\right|^{2} \mathrm{~d} \mu_{S T} \\
& \leqslant \int_{0}^{\pi} \mathrm{d} \mu_{S T} \times \int_{0}^{\pi}\left|X_{n}(\theta)\right|^{2} \mathrm{~d} \mu_{S T}=1 .
\end{aligned}
$$

It is also clear using the definition of $X_{n}(\theta)$ that the $n$-th coefficient is zero as soon as $n+2>2 L$.

We now come to $B_{L}(x)$, which is a sum of $\omega$ product functions, as already indicated: we have

$$
B_{L}(x)=\sum_{p \mid P} \beta_{L}\left(x_{p}\right) \prod_{\substack{q \mid P \\ q \neq p}} \alpha_{L}\left(x_{q}\right),
$$


where $\beta_{L}(x)$ is another trigonometric polynomial of degree $L$, given explicitly by

$$
\begin{aligned}
\beta_{L}(x) & =\frac{1}{2 L+2}\left(\sum_{|\ell| \leqslant L}\left(1-\frac{|\ell|}{L+1}\right) e(\ell x)+\sum_{|\ell| \leqslant L}\left(1-\frac{|\ell|}{L+1}\right) e(\ell(x-1 / 2))\right) \\
& =\frac{1}{2 L+2}\left(2+2 \sum_{1 \leqslant \ell \leqslant L}\left(1-\frac{\ell}{L+1}\right)\left(1+(-1)^{\ell}\right) \cos (2 \pi \ell x)\right),
\end{aligned}
$$

(see [2, p. 342, (2.3), p. 339]).

We now see immediately that Part (3) of the lemma is valid, and moreover, we see that $\left|\beta_{L}(x)\right| \leqslant 1$, so the same Cauchy-Schwarz argument already used for $\alpha_{L}$ implies that Part (2) holds.

To conclude, we look at the constant term in the Chebychev expansion for $B_{L}$, which is given by

$$
\omega\left(\int_{0}^{\pi} \alpha_{L}(\theta / \pi) \mathrm{d} \mu_{S T}\right)^{\omega-1} \int_{0}^{\pi} \beta_{L}(\theta / \pi) \mathrm{d} \mu_{S T}
$$

Using the expression

$$
\beta_{L}(\theta / \pi)=\frac{1}{2 L+2}\left(2+2 \sum_{1 \leqslant \ell \leqslant L}\left(1-\frac{|\ell|}{L+1}\right)\left(1+(-1)^{\ell}\right) \cos (2 \ell \theta)\right),
$$

where the second term doesn't contribute after integrating against $\sin ^{2} \theta=(1-$ $\cos 2 \theta) / 2$ (the term with $\ell=1$ is zero), we get the formula

$$
\Delta=\left(\int_{0}^{\pi} \alpha_{L}(\theta / \pi) \mathrm{d} \mu_{S T}\right)^{\omega-1}\left(\int_{0}^{\pi} \alpha_{L}(\theta / \pi) \mathrm{d} \mu_{S T}-\frac{\omega}{L+1}\right)
$$

for the contribution of $A_{L}(x)-B_{L}(x)$.

Now we come back to a lower bound for the constant term for $\alpha_{L}$. The point is that, as $L \rightarrow+\infty, \alpha_{L}$ converges in $L^{2}([0,1])$ to the characteristic function $\chi$ of $[0,1 / 2]$ : from $[2,(2.6)]$, and the definition of $\alpha_{L}$, we get

$$
\left|\chi(x)-\alpha_{L}(x)\right| \leqslant \beta_{L}(x), \quad 0 \leqslant x \leqslant 1,
$$

and from the Fourier expansion of $\beta_{L}$ we have

$$
\left\|\beta_{L}\right\|_{L^{2}}^{2} \leqslant \frac{1}{(2 L+2)^{2}} \times(4 L+4) \rightarrow 0
$$

Hence, we know that

$$
\frac{2}{\pi} \int_{0}^{\pi} \alpha_{L}(\theta / \pi) \sin ^{2} \theta \mathrm{d} \theta \rightarrow \int_{0}^{\pi} \chi(\theta / \pi) \mathrm{d} \mu_{S T}=1 / 2 .
$$

For given $\varepsilon \in(0,1 / 2)$, the integral is $\geqslant(1 / 2-\varepsilon / 2)$ if $L \geqslant L_{0}$, for some constant $L_{0}$. Then, if $L+1 \geqslant 2 \varepsilon^{-1} \omega$, we derive

$$
\Delta \geqslant\left(\frac{1}{2}-\frac{\varepsilon}{2}\right)^{\omega-1}\left(\frac{1}{2}-\varepsilon\right) \geqslant\left(\frac{1}{2}-\varepsilon\right)^{\omega},
$$

which gives Part (1) of the lemma. 


\section{Proof of Theorem 4}

The simple idea of the proof of Theorem 4 is that the assumption translates to $\lambda_{f_{1}}(p) \lambda_{f_{2}}(p) \geqslant 0$ for all primes $p$ (with few exceptions). However, it is well-known from Rankin-Selberg theory that if $f_{1} \neq f_{2}$, we have

$$
\sum_{p} \frac{\lambda_{f_{1}}(p) \lambda_{f_{2}}(p)}{p^{\sigma}}=O(1) \quad(\sigma \rightarrow 1+)
$$

(see, e.g, [7, §5.12] for a survey and references; the underlying fact about automorphic forms is due to Mœglin and Waldspurger). Thus we only need to find a lower bound for the left-hand side (which is a sum of non-negative terms) which is unbounded as $\sigma$ tends to $1+$. Since Rankin-Selberg theory also gives

$$
\sum_{p} \frac{\lambda_{f_{1}}(p)^{2}}{p^{\sigma}} \sim-\log (\sigma-1) \quad(\sigma \rightarrow 1+),
$$

the only difficulty is that one might fear that the coefficients of $f_{1}$ and $f_{2}$ are such that whenever $\lambda_{f_{1}}(p)$ is not small, the value of $\lambda_{f_{2}}(p)$ is very small. ${ }^{8}$ In other words, we must show that the smaller order of magnitude of (4.1) compared with (4.2) is not due to the small size of the summands, but to sign compensations. For this we use the following trick which exploits the little partial information known towards the pair Sato-Tate conjecture.

Assume first that $f_{1}$ and $f_{2}$ are non-CM cusp forms, and that neither is a quadratic twist of the other (in particular, $\left.f_{1} \neq f_{2}\right)$. By Ramakrishnan's Theorem ([18, Th. $\mathrm{M}, \S 3])$, there exists a cuspidal automorphic representation $\pi$ on $G L(4) / \mathbb{Q}$ such that

$$
L(\pi, s)=L\left(f_{1} \times f_{2}, s\right),
$$

and consequently, by Rankin-Selberg theory on $G L(4) \times G L(4)$ now (the fact that $L(\pi \times \bar{\pi}, s)$ has a single pole at $s=1)$, we have

$$
\sum_{p} \frac{\left(\lambda_{f_{1}}(p) \lambda_{f_{2}}(p)\right)^{2}}{p^{\sigma}}=\sum_{p} \frac{1}{p^{\sigma}}+O(1), \quad(\sigma \rightarrow 1+) .
$$

However, if we denote by $E$ the set of primes $p$ for which $\lambda_{f}(p) \lambda_{g}(p)<0$, we have

$$
\begin{aligned}
\sum_{p} \frac{\left(\lambda_{f_{1}}(p) \lambda_{f_{2}}(p)\right)^{2}}{p^{\sigma}} & =\sum_{p \notin E} \frac{\left(\lambda_{f_{1}}(p) \lambda_{f_{2}}(p)\right)^{2}}{p^{\sigma}}+\sum_{p \in E} \frac{\left(\lambda_{f_{1}}(p) \lambda_{f_{2}}(p)\right)^{2}}{p^{\sigma}} \\
& \leqslant 4 \sum_{p \notin E} \frac{\lambda_{f_{1}}(p) \lambda_{f_{2}}(p)}{p^{\sigma}}+16 \sum_{p \in E} \frac{1}{p^{\sigma}}
\end{aligned}
$$

by Deligne's bound. Then the first sum can also be written

$$
\begin{aligned}
4 \sum_{p \notin E} \frac{\lambda_{f_{1}}(p) \lambda_{f_{2}}(p)}{p^{\sigma}} & =-4 \sum_{p \in E} \frac{\lambda_{f_{1}}(p) \lambda_{f_{2}}(p)}{p^{\sigma}}+\sum_{p} \frac{\lambda_{f_{1}}(p) \lambda_{f_{2}}(p)}{p^{\sigma}} \\
& \leqslant 16 \sum_{p \in E} \frac{1}{p^{\sigma}}+O(1)
\end{aligned}
$$

using once more Deligne's bound and the assumption $f_{1} \neq f_{2}$ to apply (4.1).

\footnotetext{
${ }^{8}$ See the remark after the proof for an example of which potential situations must be excluded.
} 
Comparing (4.3) with these two inequalities leads to

$$
\sum_{p \in E} \frac{1}{p^{\sigma}} \geqslant \frac{1}{32} \sum_{p} \frac{1}{p^{\sigma}}+O(1), \quad \sigma \rightarrow 1,
$$

i.e., the set of primes where the signs of $f_{1}$ and $f_{2}$ differ has analytic density $\geqslant 1 / 32$.

There remains to consider Part (1) of Theorem 4 when one of the forms is of CM type (and the exceptional set $E$ now has density 0 ). We will be brief since there are less difficulties here. The main point is the following well-known result concerning the distribution of the angles $\theta_{f}(p)$ for a $\mathrm{CM}$ form $f \in \mathrm{H}_{k}^{*}(N)$, with $k \geqslant 2$ : there exists a real, non-trivial, primitive Dirichlet character $\chi_{f}$ such that $\lambda_{f}(p)=0$ when $\chi_{f}(p)=-1$ (a set of primes $I_{f}$ of density $1 / 2$ ), and for $p \notin I_{f}$, the $\theta_{f}(p) \in[0, \pi]$ for $p \leqslant x$ become uniformly distributed as $x \rightarrow+\infty$, i.e., we have

$$
\frac{2}{\pi(x)} \sum_{\substack{p \notin I_{f} \\ p \leqslant x}} e^{2 i m \theta_{f}(p)} \rightarrow 0
$$

for all non-zero integers $m \in \mathbb{Z}$ (see, e.g., [17, p. 197], where this is explained for elliptic curves, with slightly different notation). In particular, for any $\alpha>0$, the density of the set of primes where $\left|\lambda_{f}(p)\right|>\alpha$ exists and is equal to

$$
\frac{1}{\pi} \arccos (\alpha / 2)
$$

and this density goes to $1 / 2$ as $\alpha \rightarrow 0$.

Now assume $f_{1}$ is a CM form and $f_{2}$ is not; according to Lemma 4.1 below, we find $\alpha>0$ and a set of primes $P_{2}$ of analytic density $\delta>1 / 2$ where $\left|\lambda_{f_{2}}(p)\right|>\alpha$, and then the set $P_{2} \cap I_{f_{1}}$ has analytic density $>0$, thus for small enough $\alpha^{\prime}$, it contains a set $G$ with positive analytic density where $\left|\lambda_{f_{1}}(p)\right|>\alpha^{\prime}$. Hence we have

$$
\begin{aligned}
\sum_{p} \frac{\lambda_{f_{1}}(p) \lambda_{f_{2}}(p)}{p^{\sigma}} & \geqslant \sum_{p \in G} \frac{\lambda_{f_{1}}(p) \lambda_{f_{2}}(p)}{p^{\sigma}}+o\left(\log |\sigma-1|^{-1}\right) \\
& \geqslant \alpha \alpha^{\prime} \sum_{p \in G} \frac{1}{p^{\sigma}}+o\left(\log |\sigma-1|^{-1}\right), \quad \text { as } \sigma \rightarrow 1+,
\end{aligned}
$$

which is in fact a contradiction (since $f_{1}$ can not be equal to $f_{2}$ ).

Finally, assume $f_{1}$ and $f_{2}$ are CM forms. Because of independence of primitive real characters, the union $I_{f_{1}} \cup I_{f_{2}}$ has density at most $3 / 4$ (the complement contains the set of primes totally split in a Galois extension of $\mathbb{Q}$ of degree at most 4). For small enough $\alpha>0$, the complement must contain a set of primes of positive analytic density where $\left|\lambda_{f_{1}}(p)\right|>\alpha,\left|\lambda_{f_{2}}(p)\right|>\alpha$, and we can conclude as before that the Rankin-Selberg convolution has a pole at $s=1$, so that $f_{1}=f_{2}$ in that case also.

Remark 3. In the first version of this paper, we did not use Ramakrishnan's theorem, but managed to prove a weaker version of Part (1) of Theorem 4 using only the Rankin-Selberg properties of $f_{1}$ and $f_{2}$ together with the analytic properties of (small) symmetric square $L$-functions. We sketch the argument, since this may be of interest in other contexts.

The basic point is the following lemma, which may be of independent interest: 
Lemma 4.1. Let $N \geqslant 1$ be an integer, $k \geqslant 2$ be an even integer and $f \in \mathrm{H}_{k}^{*}(N)$ a primitive cusp form of level $N$ and weight $k$ which is not of CM type. Then there exists a constant $\alpha>0$ and $\delta>\frac{1}{2}$ such that

$$
\sum_{\left|\lambda_{f}(p)\right|>\alpha} \frac{1}{p^{\sigma}} \geqslant \delta \sum_{p} \frac{1}{p^{\sigma}}+O(1)
$$

for $\sigma>1$. In fact, one can take $\alpha=0.231$ and $\delta=\frac{1}{2}+\frac{1}{24}$.

Proof. It is convenient here to work with the Chebychev polynomials $U_{n}$ instead of the Chebychev functions $X_{n}$ considered in the previous section: recall that for $n \geqslant 0$, we have

$$
X_{n}(\theta)=U_{n}(2 \cos \theta)
$$

where $U_{n} \in \mathbb{R}[x]$ is a polynomial of degree $n$. Then (3.6) gives $U_{n}\left(\lambda_{f}(p)\right)=\lambda_{f}\left(p^{n}\right)$ for any $f \in \mathrm{H}_{k}^{*}(N), p \nmid N$, and $n \geqslant 0$.

We then claim that there exists a polynomial

$$
Y=\beta_{0}+\beta_{2} U_{2}+\beta_{4} U_{4}+\beta_{6} U_{6} \in \mathbb{R}[x]
$$

with the following properties:

(i) $\beta_{0}>\frac{1}{2}$

(ii) for some $\alpha>0$ and $x \in[-2,2]$, we have

$$
Y(x) \leqslant \chi_{A}(x)
$$

where $A:=\{x \in[-2,2]|| x \mid>\alpha\}$.

Assuming this, we conclude as follows: by (ii), we have

$$
\sum_{\left|\lambda_{f}(p)\right|>\alpha} \frac{1}{p^{\sigma}} \geqslant \sum_{p \nmid N} \frac{Y\left(\lambda_{f}(p)\right)}{p^{\sigma}}=\beta_{0} \sum_{p \nmid N} \frac{1}{p^{\sigma}}+\sum_{1 \leqslant i \leqslant 3} \beta_{2 i} \sum_{p \nmid N} \frac{U_{2 i}\left(\lambda_{f}(p)\right)}{p^{\sigma}} .
$$

By the holomorphy and non-vanishing at $s=1$ of the second, fourth and sixth symmetric power $L$-functions (see [10, Th. 3.3.7, Prop. 4.3] for the last two, noting that non-CM forms are not dihedral, and [22] for a survey concerning those $L$ functions), since $U_{n}\left(\lambda_{f}(p)\right)$ is exactly the $p$-th coefficient of the $n$-th symmetric power for $p \nmid N$, standard analytic arguments show that

$$
\sum_{p \nmid N} \frac{U_{2 i}\left(\lambda_{f}(p)\right)}{p^{\sigma}}=O(1)
$$

for $\sigma \geqslant 1$ and $i=1,2,3$. Hence the result follows with $\delta=\beta_{0}>\frac{1}{2}$.

Now to check the claim, and verify the values of $\alpha$ and $\delta$, we just exhibit a suitable polynomial, namely

since

$$
Y=\frac{1}{2}+\frac{1}{24}+\frac{1}{4} U_{2}-\frac{1}{4} U_{4}+\frac{136}{1000} U_{6}=\frac{17}{125} x^{6}-\frac{93}{100} x^{4}+\frac{227}{125} x^{2}-\frac{283}{3000},
$$

$$
\left\{\begin{array}{l}
U_{0}=1, \quad U_{1}=x, \quad U_{2}=x^{2}-1, \quad U_{4}=x^{4}-3 x^{2}+1 \\
U_{5}=x^{5}-4 x^{3}+3 x, \quad U_{6}=x^{6}-5 x^{4}+6 x^{2}-1 .
\end{array}\right.
$$

This polynomial is even, and its graph on $[-2,2]$ is in Figure 1.

The value of $\alpha$ is an approximation (from below) to the real root

$$
\alpha_{0}=0.23107202470801418176315245050693402580 \ldots
$$




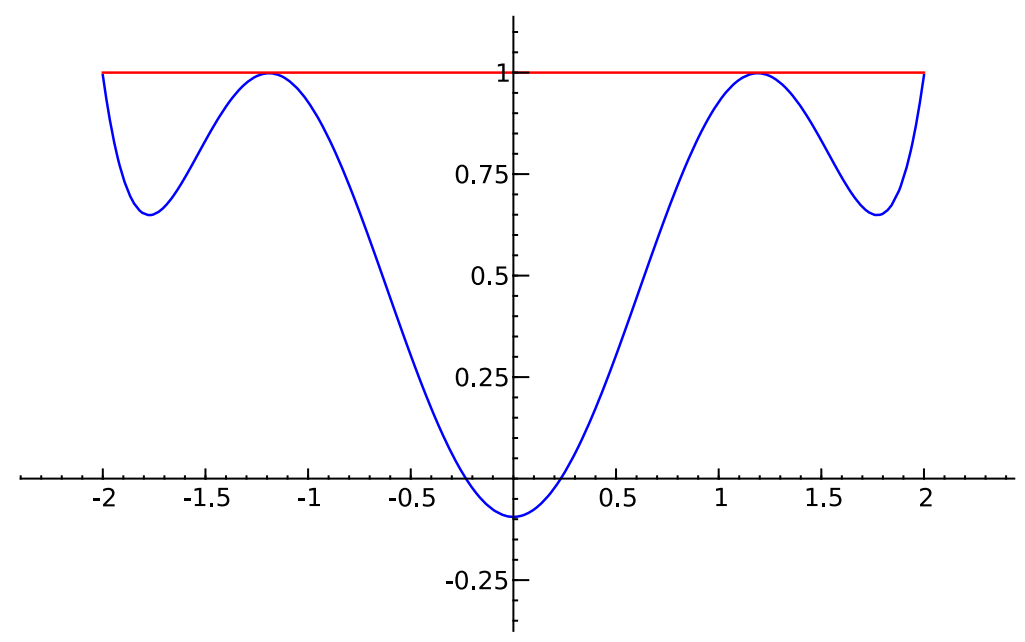

FiguRe 1

of $Y$ in $[0,2]$; the maximum value of $Y$ on $[0,2]$ is very close to 1 .

The upshot of this lemma is that, given $f_{1}$ and $f_{2}$ (not of CM type), there exists a set of primes of analytic density $>0$ for which both $\left|\lambda_{f_{1}}(p)\right|$ and $\left|\lambda_{f_{2}}(p)\right|$ have a positive lower bound. Then the sum of $\lambda_{f_{1}}(p) \lambda_{f_{2}}(p)$ over this set can not be small, and this leads to an upper bound for the density of the "exceptional set". However, the actual value from the above lemma is much smaller than what Theorem 4 uses (it is about $1 / 1000$ ).

Another interesting point of this method is that using the sixth symmetric power (and thus the deep results of Kim and Shahidi) is necessary for Lemma 4.1. For this, note that the sequences $\left\{x_{p}\right\}_{p \text { primes }}$ and $\left\{y_{p}\right\}_{p \text { primes }}$ defined by $x_{2}=y_{2}=0$ and for primes $p \geqslant 3$ by

$$
\begin{aligned}
& x_{p}= \begin{cases}0 & \text { if } p \equiv 3(\bmod 4), \\
(-1)^{(p-1) / 4} \sqrt{2} & \text { if } p \equiv 1(\bmod 4),\end{cases} \\
& y_{p}= \begin{cases}(-1)^{(p-3) / 4} \sqrt{2} & \text { if } p \equiv 3(\bmod 4), \\
0 & \text { if } p \equiv 1(\bmod 4),\end{cases}
\end{aligned}
$$

have the "right" moments of order 1 to 5 for being Sato-Tate distributed, ${ }^{9}$ i.e., we have

$$
\sum_{p} \frac{X_{k}\left(x_{p}\right)}{p^{\sigma}}=O(1) \quad \text { and } \quad \sum_{p} \frac{X_{k}\left(y_{p}\right)}{p^{\sigma}}=O(1)
$$

for $\sigma>1$ and $1 \leqslant k \leqslant 5$, and yet $x_{p} y_{p} \geqslant 0$ for all $p$, in fact $x_{p} y_{p}=0$, so that we most certainly have

$$
\sum_{p} \frac{x_{p} y_{p}}{p^{\sigma}}=O(1) \quad(\sigma>1)
$$

Remark 4. As a final remark, one can think of other ways (than looking at signs) of reducing Fourier coefficients of modular forms to a fixed finite set: the most obvious, at least if $f$ has integral coefficients $\lambda_{f}(n) n^{(k-1) / 2}$, is to look at the coefficients modulo

\footnotetext{
9 The sixth moment fails: it is 4 instead of 5 for the Sato-Tate distribution.
} 
some fixed prime number $\ell$. However, the situation there can be drastically different: for instance, for all (infinitely many) elliptic curves with full rational 2-torsion, given for instance by equations

$$
y^{2}=(x-a)(x-b)(x-c)
$$

with $a, b, c$ distinct integers, the reduction modulo 2 of the odd prime coefficients of the corresponding $L$-function (or modular form) is the same!

\section{REFERENCES}

[1] T. Barnet-Lamb, D. Geraghty, M. Harris \& R. Taylor, A family of Calabi-Yau varieties and potential automorphy II, preprint (2009), available at http://www.math.harvard.edu/ rtaylor/

[2] J. T. Barton, H. L. Montgomery \& J. D. Vaaler, Note on a diophantine inequality in several variables, Proc. AMS 129 (2001), 337-345.

[3] W. Duke \& E. Kowalski, A problem of Linnik for elliptic curves and mean-value estimates for automorphic representations, With an appendix by Dinakar Ramakrishnan, Invent. math. 139 (2000), no. 1, 1-39.

[4] D. Goldfeld \& J. Hoffstein, On the number of Fourier coefficients that determine a modular form, in: A tribute to Emil Grosswald: number theory and related analysis, Contemp. Math. 143, Amer. Math. Soc., 1993, 385-393.

[5] A. Granville and K. Soundararajan, The spectrum of multiplicative functions, Ann. of Math. 153 (2001), no. 2, 407-470.

[6] H. Iwaniec, W. Kohnen \& J. Sengupta, The first negative Hecke eigenvalue, International J. Number Theory 3 (2007), No. 3, 355-363.

[7] H. Iwaniec \& E. Kowalski, Analytic Number Theory, American Mathematical Society Colloquium Publications, 53. American Mathematical Society, Providence, RI, 2004. xii+615.

[8] H. Iwaniec, W. Luo \& P. Sarnak, Low-lying zeros of families of L-functions, Publ. Math. I.H.É.S 91 (2000), 55-131.

[9] E. Kowalski, Variants of recognition problems for modular forms, Arch. Math. (Basel) 84 (2005), No. 5, 57-70.

[10] H. Kim \& F. Shahidi, Cuspidality of symmetric powers with applications, Duke Math. J. 112 (2002), 177-197.

[11] Y. Lamzouri, The two-dimensional distribution of values of $\zeta(1+i t)$, IMRN, Vol. 2008, Article ID rnn106, 48 pages.

[12] Y.-K. Lau \& J. Wu, On the least quadratic non-residue, International J. Number Theory 4 (2008), No 3, 423-435.

[13] Y.-K. Lau \& J. Wu, A large sieve inequality of Elliott-Montgomery-Vaughan type and two applications, IMRN, Vol. 2008, Number 5, Article ID rnm 162, 35 pages.

[14] B. Mazur, Finding meaning in error terms, Bull. A.M.S 45 (2008), 185-228.

[15] P. Michel \& A. Venkatesh, The subconvexity problem for $G L_{2}$, arXiv:0903.3591v1.

[16] M. R. Murty, Congruences between modular forms, in: "Analytic number theory (Kyoto, 1996)", London Math. Soc. Lecture Note Ser. 247, Cambridge Univ. Press, 1997, 309-320.

[17] V. K. Murty, On the Sato-Tate conjecture, in Progress in Math. 26, Birkhaüser 1982, p. 195-205.

[18] D. Ramakrishnan, Modularity of the Rankin-Selberg L-series, and multiplicity one for SL(2), Ann. of Math. (2) 152 (2000), no. 1, 45-111.

$[19]$ E. Royer, Facteurs $\mathbb{Q}$-simples de $J_{0}(N)$ de grande dimension et de grand rang, Bull. Soc. Math. France 128 (2000), 219-248.

[20] P. Sarnak, Statistical properties of eigenvalues of the Hecke operators, in "Analytic Number Theory and Diophantine Problems" (Stillwater, OK, 1984), Progr. Math. 70, Birkhäuser, 1987, 321-331.

[21] J.-P. Serre, Répartition asymptotique des valeurs propres de l'opérateur de Hecke $T_{p}$, J. American Math. Soc. 10 (1997), 75-102. 
[22] F. Shahidi, Symmetric power L-functions for $G L(2)$, in: "Elliptic curves and related topics", edited by E. Kishilevsky and M. Ram Murty, CRM Proc. and Lecture Notes 4, 1994, 159-182.

[23] G. Tenenbaum, Cribler les entiers sans grand facteur premier, Philos. Trans. Roy. Soc. London Ser. A 345 (1993), no. 1676, 377-384.

[24] G. Tenenbaum, Introduction to analytic and probabilistic number theory, Cambridge Studies in Advanced Mathematics 46, Cambridge University Press, 1995.

[25] G. Tenenbaum, in collaboration with J. Wu, Exercices corrigés de théorie analytique et probabiliste des nombres, Cours spécialisés, n 2, Société Mathématique de France (1996), xiv $+251 \mathrm{pp}$.

[26] G. Tenenbaum and $\mathrm{J} . \mathrm{Wu}$, Moyennes de certaines fonctions multiplicatives sur les entiers friables, J. reine angew. Math. 564 (2003), 119-166.

ETH ZÜrich - D-MATH, RÄmistrasse 101, 8092 Zürich, Switzerland

E-mail address: kowalski@math.ethz.ch

Department of Mathematics, The University of Hong Kong, Pokfulam Road, HONG KONG

E-mail address: yklau@maths.hku.hk

Department of Mathematics, Stanford University, Stanford, CA 94305, USA

E-mail address: ksound@stanford.edu

Institut Elie Cartan Nancy (IECN), CNRS, NAnCy-Université, INRIA, Boulevard des Aiguillettes, B.P. 239, 54506 Vandeuvre-lès-Nancy, France

E-mail address: wujie@iecn.u-nancy.fr 